\title{
Ultrastable Plasmonic Bioink for Printable Point-of-Care Biosensors
}

Ze Yin ${ }^{\dagger a}$, Heng Guo ${ }^{\dagger^{a}}$, Yixuan Li ${ }^{a}$, Joshua Chiu ${ }^{a}$ Limei Tian ${ }^{* a}$

${ }^{a}$ Department of Biomedical Engineering, and Center for Remote Health Technologies and Systems, Texas A\&M University, College Station, TX 77843, USA

* Corresponding Author: Dr. Limei Tian, Email: $\underline{\text { tian@tamu.edu }}$

$\dagger$ These authors contributed equally 

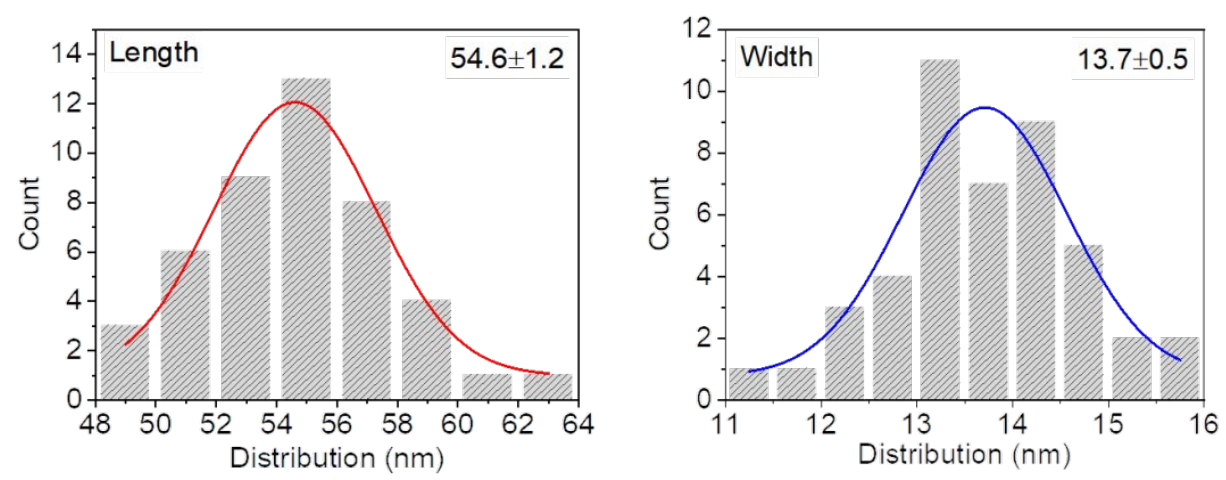

Figure S1. Size distributions of the as-prepared AuNRs exhibit good size uniformity.

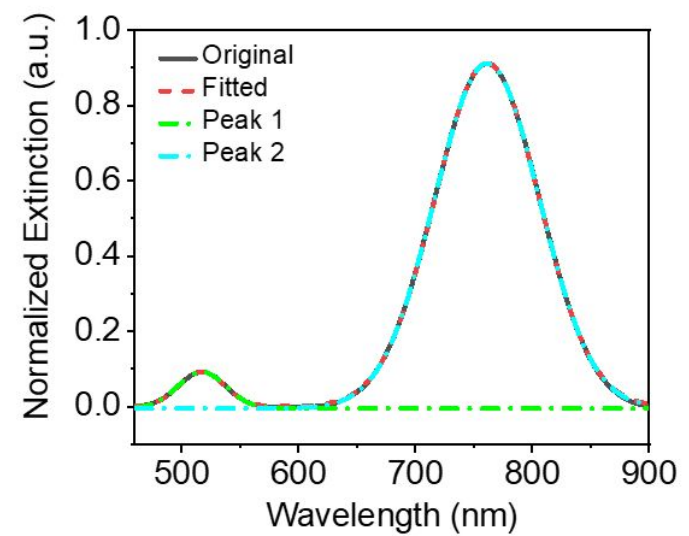

Figure S2. Representative fitted extinction spectra for the determination of LSPR peak position.

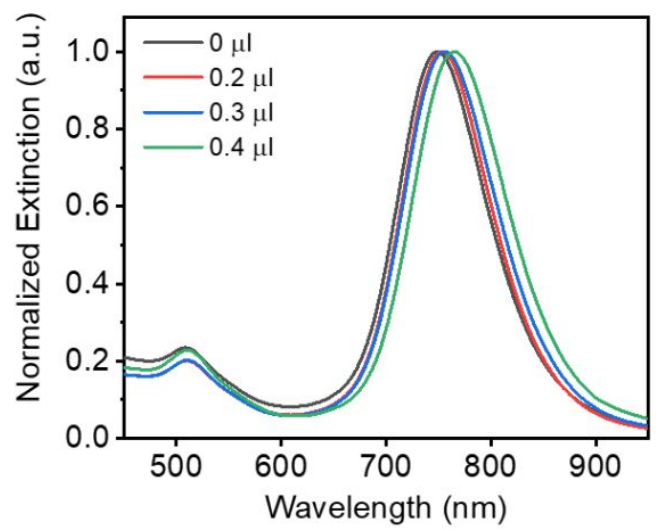

Figure S3. Normalized extinction spectra of the plasmonic bioink prepared with different volumes of the TMPS and APTMS solution, indicating the increase in the thickness of the encapsulation layer. 

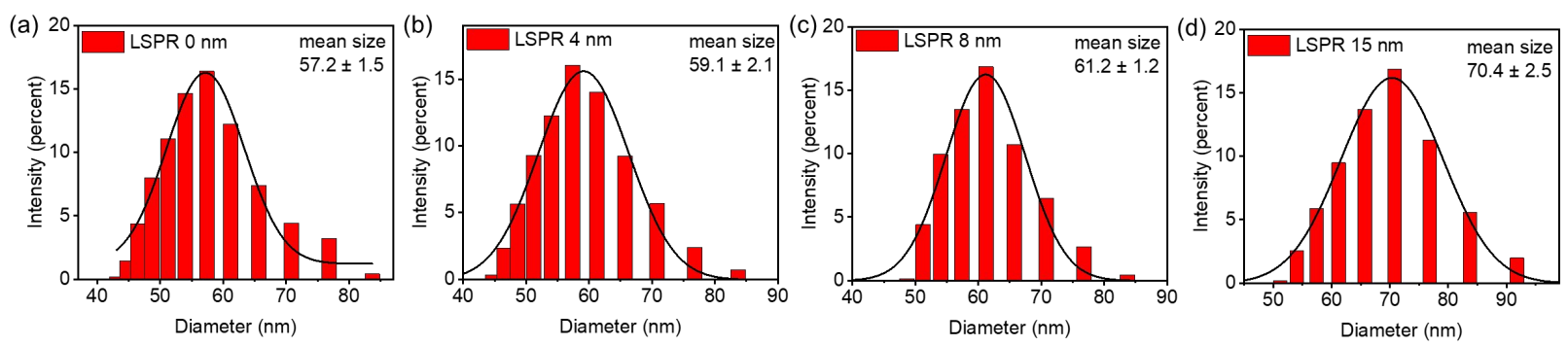

Figure S4. The hydrodynamic diameters of (a) AuNRs-PA-IgGs and (b-d) encapsulated AuNRsPA-IgGs measured with dynamic light scattering (DLS) corresponding to the incremental red shifts of (b) $4 \mathrm{~nm}$, (c) $8 \mathrm{~nm}$ and (d) $15 \mathrm{~nm}$ in the LSPR wavelength of the encapsulated AuNRsPA-IgG bionanoconjugates.

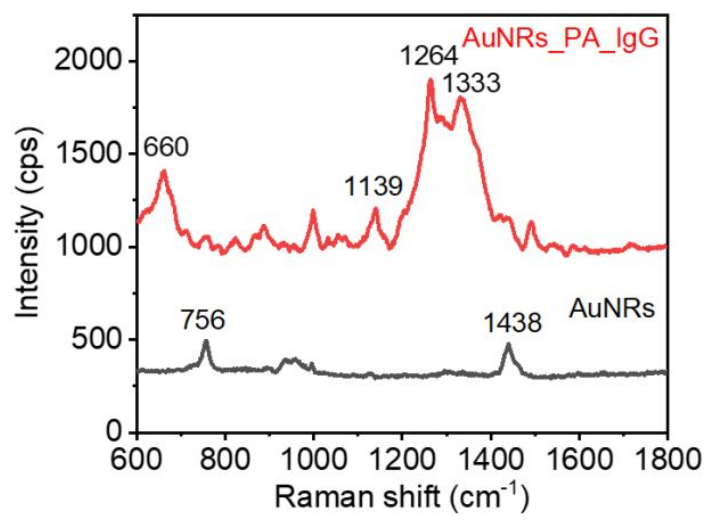

Figure S5. Raman spectra of AuNRs before and after IgG immoblization indicating the successful IgG immoblization on the surface of AuNRs.

(a)

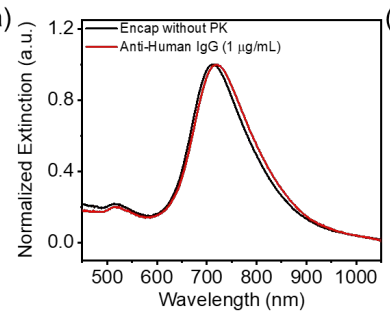

(e)

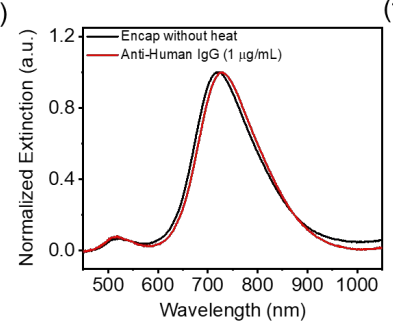

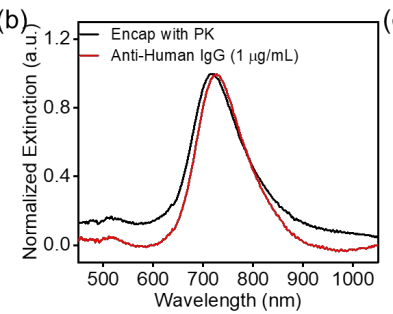

(f)

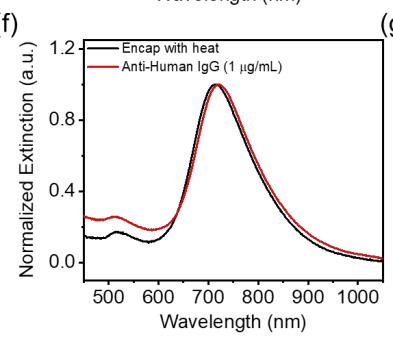

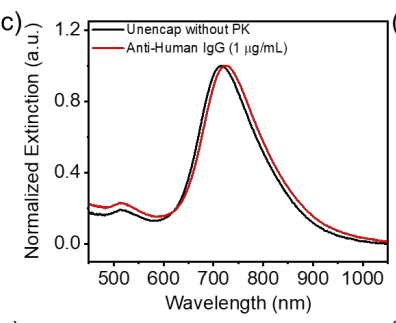

(g)

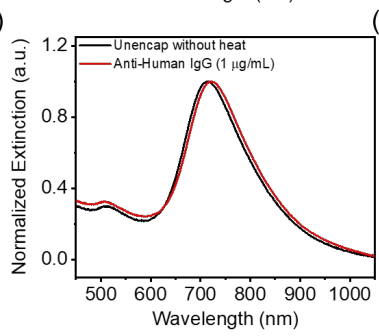

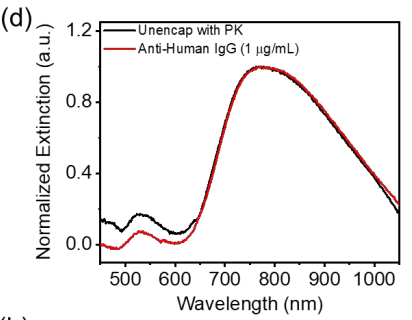

(h)

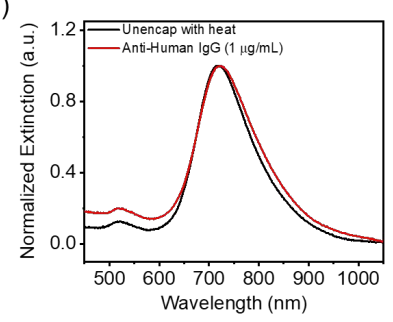


Figure S6. Normalized extinction spectra of the plasmonic biochips prepared with the bioinks of encapsulated human $\operatorname{IgG}(\mathrm{a})$ without and (b) with proteinase $\mathrm{K}$ treatment before and after exposure to anti-human IgG. Normalized extinction spectra of the plasmonic biochips prepared with the bioinks of unencapsulated human $\operatorname{IgG}(\mathrm{c})$ without and (d) with proteinase $\mathrm{K}$ treatment before and after exposure to anti-human IgG. Normalized extinction spectra of the plasmonic biochips prepared with the bioinks of encapsulated human $\operatorname{IgG}(\mathrm{e})$ without and (f) with thermal treatment before and after exposure to anti-human IgG. Normalized extinction spectra of the plasmonic biochips prepared with the bioinks of unencapsulated human $\operatorname{IgG}(\mathrm{g})$ without and $(\mathrm{h})$ with thermal treatment before and after exposure to anti-human IgG. Anti-human IgG concentration is $1 \mu \mathrm{g} / \mathrm{mL}$.
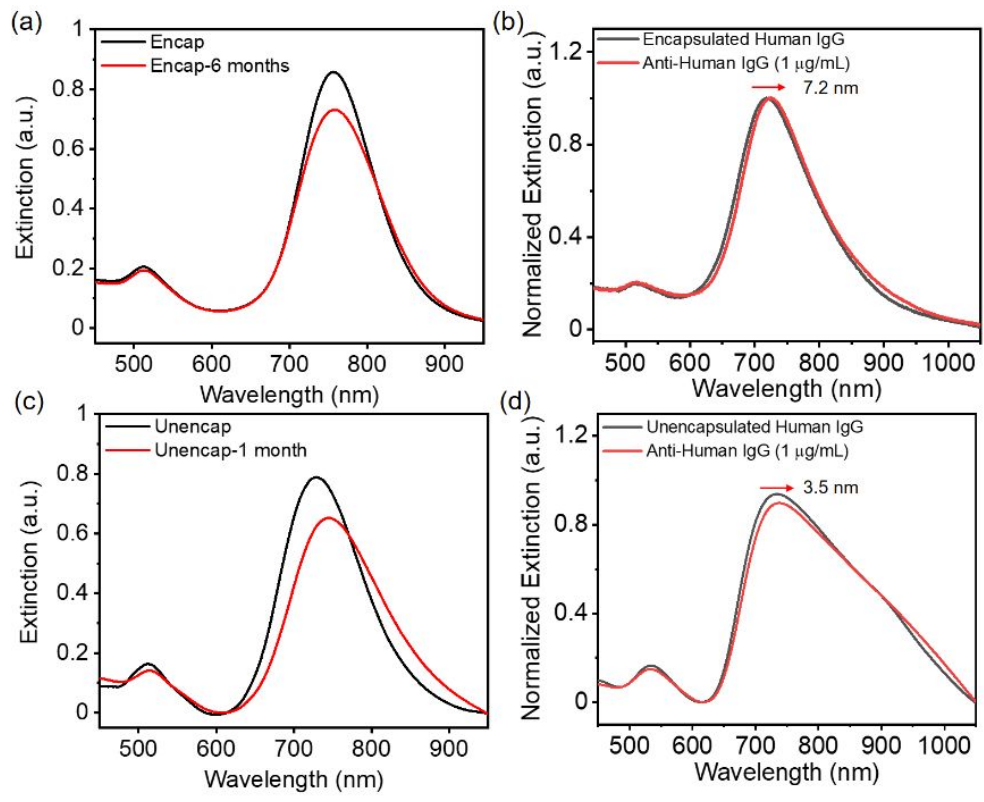

Figure S7. (a) Extinction spectra of encapsulated AuNR-PA-IgG bioink before and after storage stored at $4{ }^{\circ} \mathrm{C}$ for 6 months. (b) Extinction spectra of the 6-month-old encapsulated AuNR-PA-IgG on a glass slide after exposure to anti-IgG of $1 \mu \mathrm{g} / \mathrm{mL}$. (c) Extinction spectra of unencapsulated AuNR-PA-IgG bioink before and after storage stored at $4{ }^{\circ} \mathrm{C}$ for 1 month. (b) Extinction spectra of the 1-month-old unencapsulated AuNR-PA-IgG on a glass slide after exposure to anti-IgG of 1 $\mu \mathrm{g} / \mathrm{mL}$. 
(a)

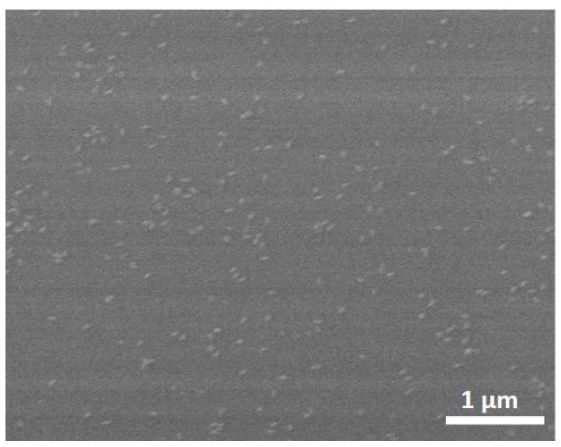

(b)

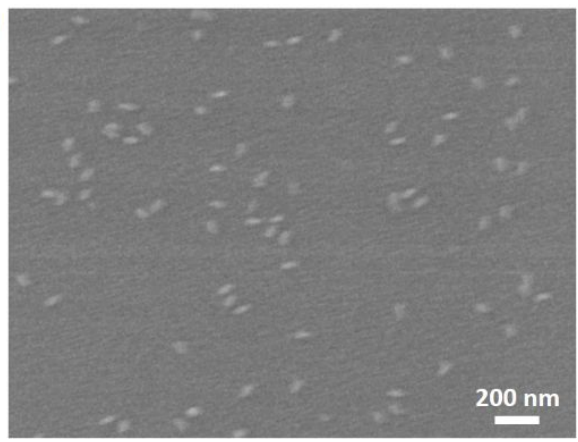

Figure S8. Scanning electron microscopy (SEM) images of the plasmonic bioink deposited on a PSS modified glass substrate show uniform distribution of AuNR.

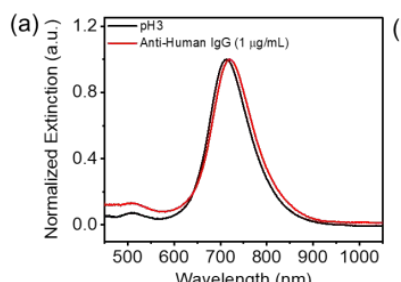

(e)

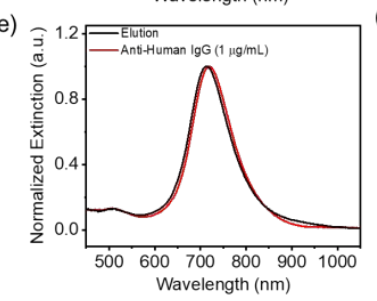

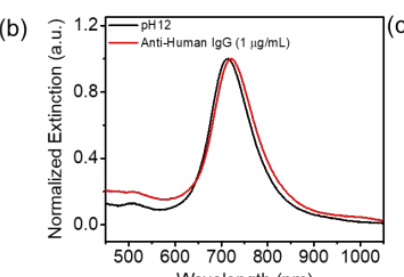

(1)

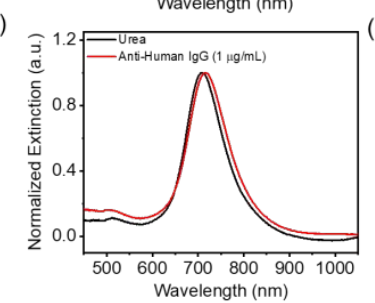

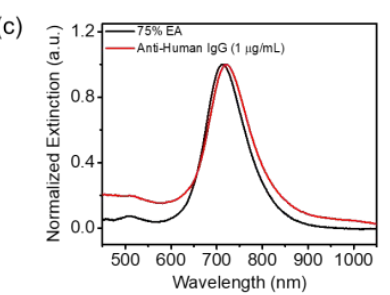

(g)

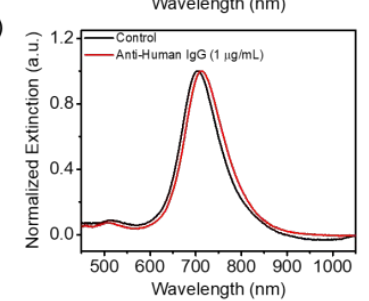

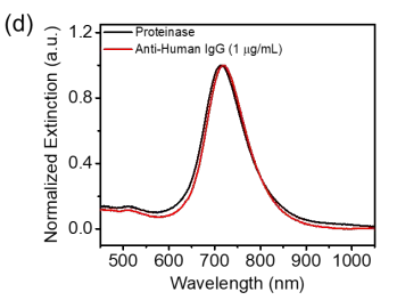

Wavelength $(\mathrm{nm})$

Figure S9. (a-g) Normalized extinction spectra of the plasmonic biochips with encapsulated AuNR-PA-IgG, and after exposing to a wide range of chemical and biological denaturants and then exposing to anti-IgG of $1 \mu \mathrm{g} / \mathrm{mL}$.
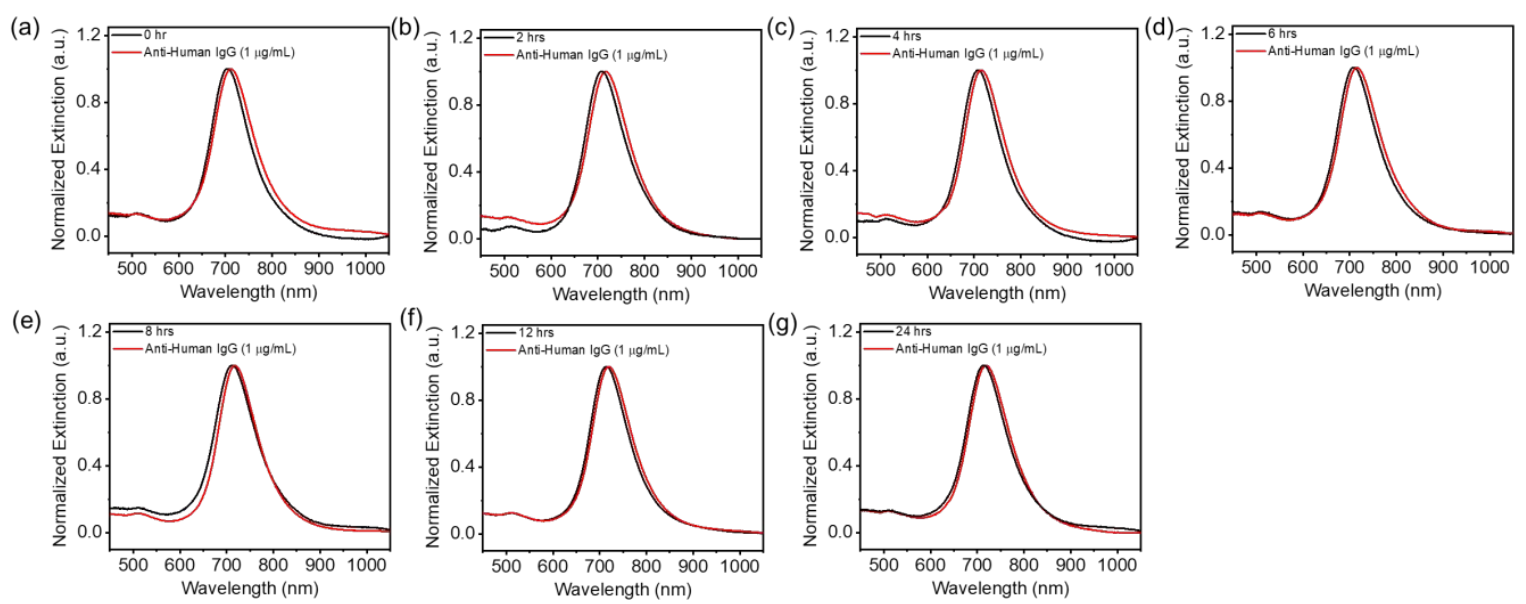

Figure S10. (a-g) Normalized extinction spectra of the plasmonic biochips with encapsulated 
AuNR-PA-IgG, and after exposing to $60^{\circ} \mathrm{C}$ for different durations and then exposing to anti-IgG of $1 \mu \mathrm{g} / \mathrm{mL}$.
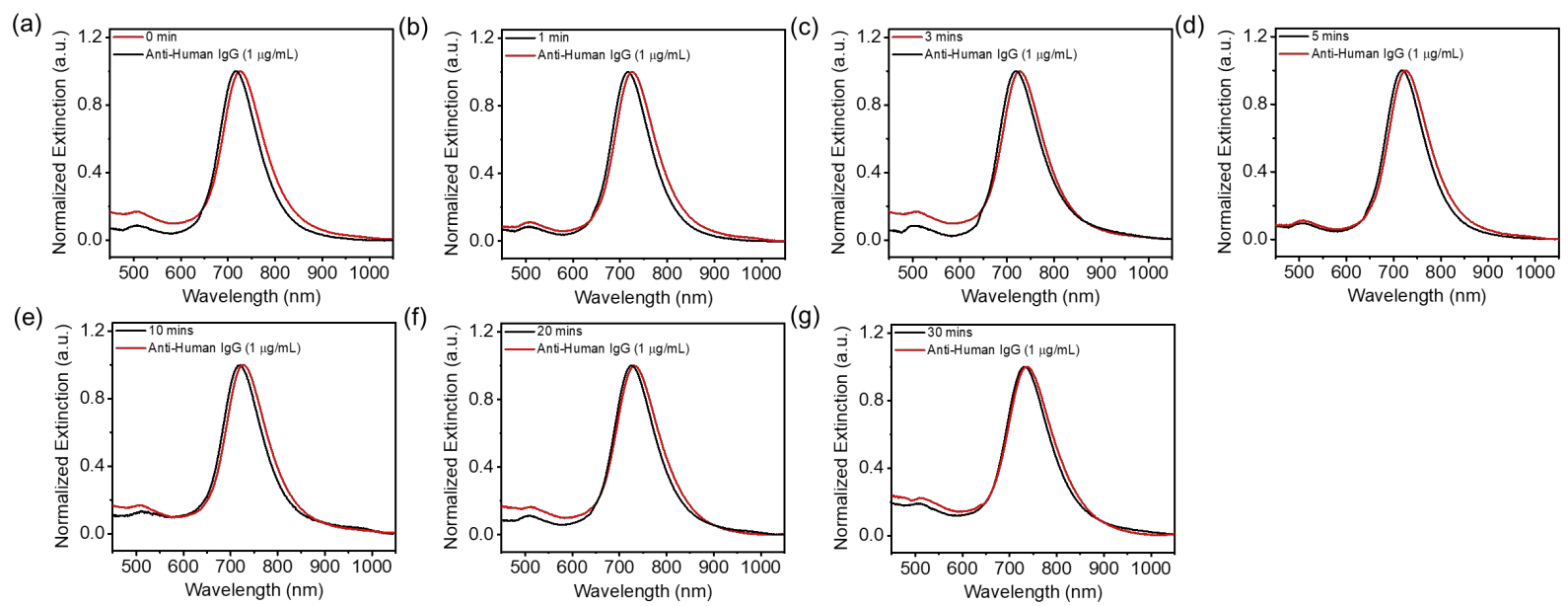

Figure S11. (a-g) Normalized extinction spectra of the plasmonic biochips with encapsulated AuNR-PA-IgG, and after exposing to ultrasonic agitation for different durations and then exposing to anti-IgG of $1 \mu \mathrm{g} / \mathrm{mL}$.
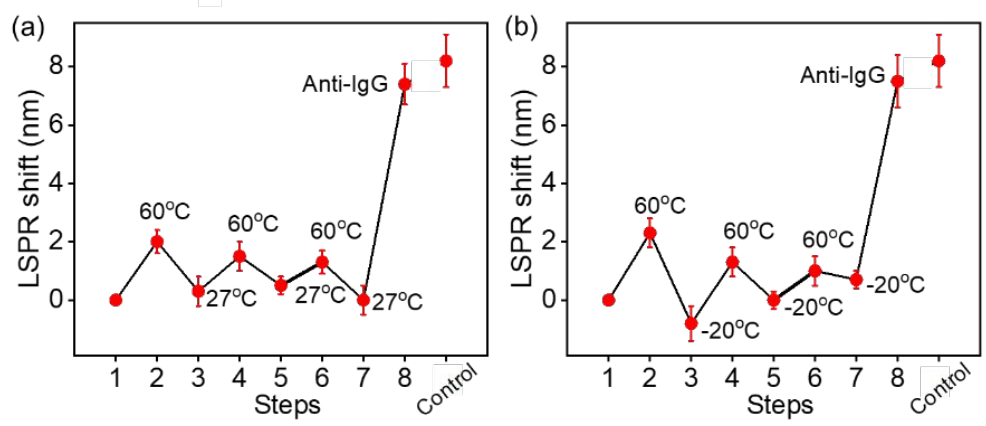

Figure S12. LSPR shifts corresponding to temperature fluctuations and the binding of anti-human IgG of $1 \mu \mathrm{g} / \mathrm{mL}$. Control represents the LSPR wavelength shift of plasmonic biochips with encapsulated AuNR-PA-IgG without exposure to temperature fluctuations after the binding of anti-human IgG of $1 \mu \mathrm{g} / \mathrm{mL}$. Error bars represent standard deviations from three replicates. 


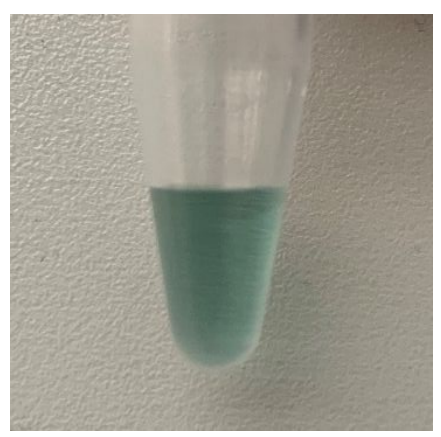

Figure S13. Photograph of the encapsulated plasmonic bioink in a $1.5 \mathrm{ml}$ centrifuge tube. For visualizaton, the AuNRs with the longitudinal LSPR wavelength at $660 \mathrm{~nm}$ is used.
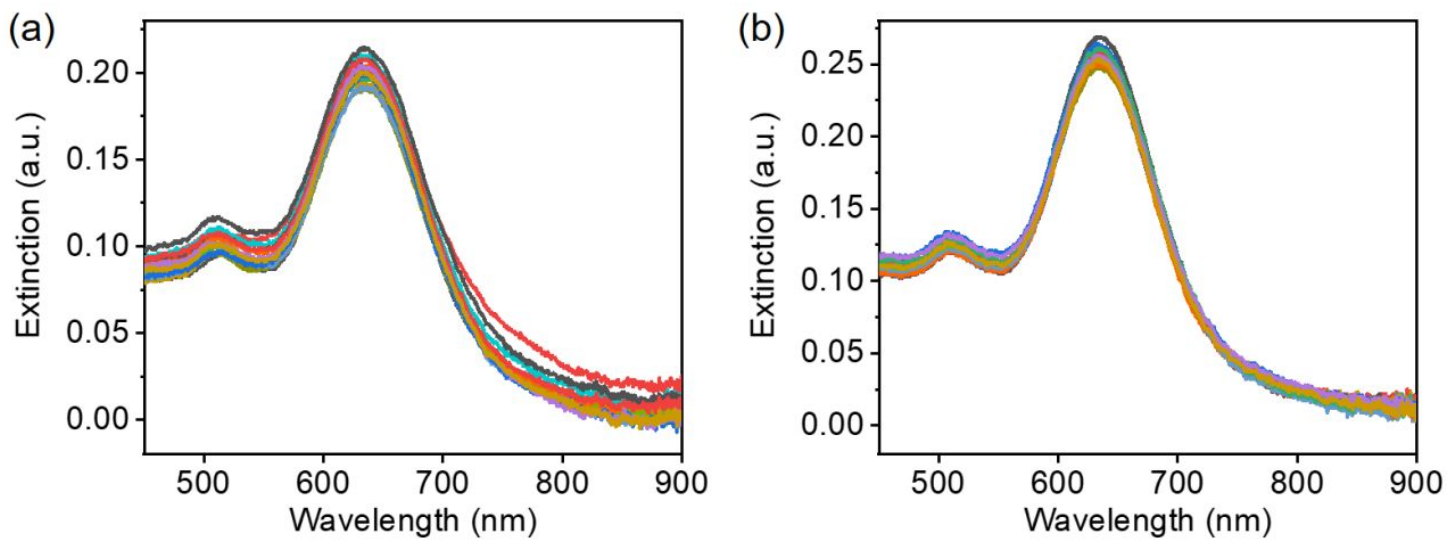

Figure S14. Extinction spectra of the printed AuNRs on a nitrocellulose membrane, collected from 18 different positions of (a) lines and (b) dots (6 spectra per line/dot) showing the excellent spectral uniformity of the printed patterns.

(a)

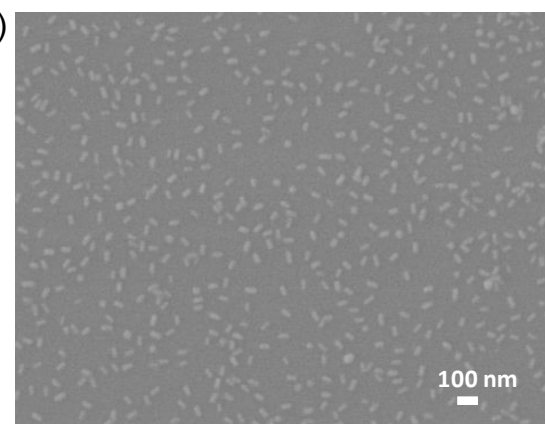

(b)

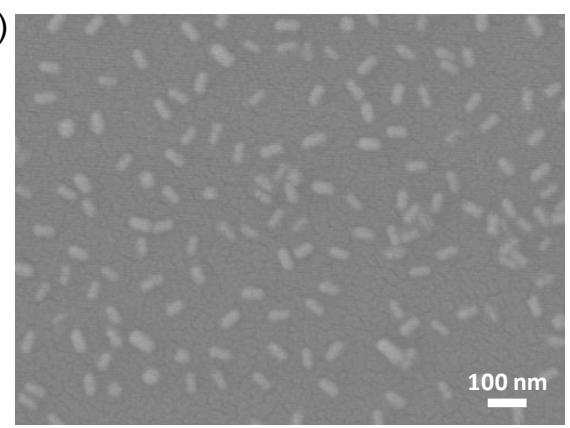

Figure S15. Scanning electron microscopy (SEM) images of the plasmonic bioink deposited on a PSS modified silicon substrate show the uniform distribution of AuNRs. 